\title{
PAX6, a novel target of miR-335, inhibits cell proliferation and invasion in glioma cells
}

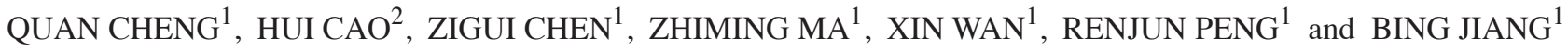 \\ ${ }^{1}$ Department of Neurosurgery, Xiangya Hospital of Central South University, Changsha, Hunan 410008; \\ ${ }^{2}$ Department of Neurosurgery, Brain Hospital of Hunan Province, The Affiliated Hospital of \\ Hunan Traditional Chinese Medicine University, Changsha, Hunan 410007, P.R. China
}

Received September 22, 2013; Accepted March 17, 2014

DOI: $10.3892 / \mathrm{mmr} .2014 .2150$

\begin{abstract}
Paired box 6 (PAX6), a highly conserved transcription factor, is important in glioma. However, the molecular mechanisms involved remain unclear. The present study demonstrated that the expression of PAX6 was significantly reduced with the malignancy of glioma and also identified PAX6 as a novel target of microRNA (miR)-335, which was significantly upregulated in glioma. The inhibition of miR-335 increased the protein expression of PAX6, whereas the upregulation of miR-335 suppressed its expression in human glioma U251 and U87 cells. Furthermore, upregulation of miR-335 promoted U251 cell proliferation, colony formation and invasion, which was reversed by the overexpression of PAX6. Furthermore, the present study demonstrated that the effect of miR-335 on U251 cell invasion was via the modulation of matrix metalloproteinase (MMP)-2 and MMP-9 expression by targeting PAX6. In conclusion, the present study demonstrated that PAX6, as a novel target of miR-335, has an anti-oncogenic function in glioma, and thus PAX6 may serve as a therapeutic target for glioma.
\end{abstract}

\section{Introduction}

Paired box 6 (PAX6) is a highly conserved transcription regulatory factor involved in multiple biological processes, including embryogenesis, cell differentiation, biosynthesis and cell motility $(1,2)$. Accumulating evidence has demonstrated that PAX6 is crucial in the development of the central nervous system (3). Previously, several studies have suggested that PAX6 acts as a tumor suppressor in malignant glioma $(4,5)$. As one of the most common types of primary brain cancer, the prognosis of malignant glioma is very poor, mainly due

Correspondence to: Professor Bing Jiang, Department of Neurosurgery, Xiangya Hospital of Central South University, 87 Xiangya Road, Changsha, Hunan 410008, P.R. China

E-mail: csubingjiang@163.com

Key words: glioma, paired box 6, microRNA-335, proliferation, invasion to its resistance to radiotherapy, chemotherapy or adjuvant therapies (6-8). The median survival rate of glioma has not improved oyer the last decade, despite the development of therapeutic strategies for various types of human cancer (9). Since PAX6 has been suggested to act as a suppressive regulator in malignant glioma, it may be a promising therapeutic target.

MicroRNAs (miRNAs) are a type of endogenous non-coding RNA, which are able to bind to the 3'-untranslated region (3'-UTR) of its target mRNAs, eventually causing mRNA degradation or translational repression (10). In previous decades, accumulating studies have demonstrated that miRNAs act as key regulators in the development and progression of various types of cancer, including malignant glioma (11). For instance, miR-155 was reported to regulate cell proliferation and invasion in glioma (12). miR-128 was demonstrated to promote cell-cell adhesion in glioma U87 cells (13). Therefore, miRNAs are potential therapeutic targets or candidates for the treatment of glioma.

miR-335 has been demonstrated to be implicated in multiple types of malignant tumor, including small cell lung cancer, osteosarcoma, ovarian cancer, prostate cancer, hepatocellular carcinoma, meningioma and gastric cancer (14-22). Recently, Jiang et al have suggested that miR-335 upregulation is associated with advanced tumor progression in glioma and is an independent marker for the predication of the clinical outcome of patients with glioma (23). However, the exact regulatory roles of miR-335 and PAX6, as well as their association with glioma, have never been studied.

The present study investigated the expression of PAX6 and miR-335 in glioma tissues, as well as their effects on glioma cell proliferation, colony formation and invasion in vitro. Furthermore, the present study also investigated the involved molecular mechanisms and unveiled their target association.

\section{Materials and methods}

Tissues and cell lines. All protocols in the present study were approved by the Ethical Committee of Central South University (Changsha, Hunan, China). In addition, all informed consent forms for patients participating in the present study were obtained. In total, 24 glioma tissues (six cases of WHO I, six cases of WHO II, six cases of WHO III and six cases of WHO IV), as well as six cases of normal brain 
tissues were obtained from the Department of Neurosurgery, Xiangya Hospital of Central South University (Changsha, Hunan, China). Human glioma U251 and U87 cell lines were obtained from the Cell Bank of Central South University (Changsha, Hunan, China). All cells were cultured in DMEM supplemented with $10 \%$ fetal bovine serum (FBS), $100 \mathrm{IU} / \mathrm{ml}$ of penicillin and $100 \mu \mathrm{g} / \mathrm{ml}$ of streptomycin sulfate at $37^{\circ} \mathrm{C}$ in a humidified incubator containing $5 \% \mathrm{CO}_{2}$.

RNA extraction and real-time RT-PCR analysis. According to the manufacturer's instructions, total RNA was extracted with TRIzol (Invitrogen Life Technologies, Carlsbad, CA, USA). For the analysis of miR-335 expression, $10 \mathrm{ng}$ of RNA was converted to cDNA using miR-335-specific primers and an ABI miRNA Reverse Transcription kit (Applied Biosystems, Foster City, CA, USA). Following that, real-time PCR was performed on an ABI 7500 thermocycler (Applied Biosystems). The U6 gene was used as an internal reference. For the analysis of PAX6 expression, a TaqMan Reverse Transcription kit and Power SYBR-Green kit (Thermo Fisher Scientific, Waltham, MA, USA) were used to perform real-time RT-PCR, and $\beta$-actin was used as an internal reference. The relative expression was analyzed by the $2^{-\Delta \Delta C t}$ method. The primers used were as follows: PAX6, forward 5'-AACGATAACATACCAAGCGTGT-3' and reverse 5'-GGTCTGCCCGTTCAACATC-3'; $\beta$-actin, forward 5'-AGGGGCCGGACTCGTCATACT-3' and reverse 5'-GGCGGCACCACCATGTACCCT-3'.

Western blot analysis. Tissues or cells were solubilized in cold RIPA lysis buffer. Proteins were separated with $10 \%$ SDS-PAGE and then transferred onto a polyvinylidene difluoride (PVDF) membrane, which was then incubated with TBST containing 5\% skimmed milk at room temperature for $4 \mathrm{~h}$, and then with the primary antibodies of anti-PAX6, anti-MMP-2, anti-MMP-9 and anti- $\beta$-actin (Abcam, Cambridge, UK), respectively, at room temperature for $3 \mathrm{~h}$. Following being washed by PBST for $15 \mathrm{~min}$, the PVDF membrane was incubated for 40 min with the corresponding secondary antibodies. An ECL kit (Pierce Biotechnology, Inc., Rockford, IL, USA) was used to perform chemiluminescent detection. Image-Pro plus software 6.0 was used to analyze the relative protein expression, presented as the density ratio versus $\beta$-actin.

Transfection.Lipofectamine 2000(InvitrogenLife Technologies) was used to perform transfection according to the manufacturer's instructions. For the functional analysis of PAX6, cells were transfected with the PAX6-pCMV-NEO-BAM plasmid. For the functional analysis of miR-335, cells were transfected with the scrambled miRNA as a negative control (NC), the miR-335 mimics or the miR-335 inhibitor (Invitrogen Life Technologies).

Dual luciferase reporter assays. A QuikChange site-directed mutagenesis kit (Stratagene, La Jolla, CA, USA) was used to generate a wild type and a mutant type 3'-UTR of PAX6, which was then inserted into the multiple cloning sites in the psiCHECK ${ }^{\mathrm{TM}} 2$ vector (Promega, Madison, WI, USA), respectively. U87 and U251 cells were cultured to 50-60\% confluence in a 24-well plate and then co-transfected with psiCHECK ${ }^{\text {TM }}$ 2-PAX6-3'-UTR or psiCHECK ${ }^{\text {Tм }} 2$-mut PAX6-3'-UTR vector, with or without $50 \mathrm{nM}$ of miR-335, using cellfectin II reagent (Invitrogen Life Technologies). The luciferase activity for each group was determined $48 \mathrm{~h}$ after cotransfection using the dual-luciferase reporter assay system (Promega) and a Beckman Coulter LD 400 luminometer (Beckman Coulter, Fullerton, CA, USA). Renilla luciferase activity was normalized to firefly luciferase activity.

Cell proliferation assay. An MTT assay was used to measure cell proliferation. At $48 \mathrm{~h}$ post-transfection, the transfection medium in each well was replaced with $100 \mu \mathrm{l}$ of fresh serum-free medium with $0.5 \mathrm{~g} / 1$ of MTT. Following incubation at $37^{\circ} \mathrm{C}$ for $4 \mathrm{~h}$, the MTT medium was removed by aspiration and $50 \mu \mathrm{l}$ of DMSO was added to each well. Following incubation at $37^{\circ} \mathrm{C}$ for a further $10 \mathrm{~min}$, the optical density at $570 \mathrm{~nm}$ was measured using a Bio-Tek ELx800 type ELISA reader (Bio-Tek Instruments, Inc., Winooski, VT, USA). This experiment was repeated three times.

Invasion assay. The invasive assay was performed in 24-well transwell chambers (Chemicon, Temecula, CA, USA) with a layer of matrigel. According to the manufacturer's instructions, $200 \mu \mathrm{l}$ of cell suspension $\left(1 \times 10^{6}\right.$ cells $\left./ \mathrm{ml}\right)$ was added in triplicate wells for each group, which was then incubated in a humidified atmosphere of $5 \% \mathrm{CO}_{2}$ at $37^{\circ} \mathrm{C}$ for $24 \mathrm{~h}$. The cells that had migrated through the filter were stained with gentian violet. Following being washed with PBS for $15 \mathrm{~min}$, five fields were randomly selected under the microscope and the cell number was counted.

Statistical analysis. Statistical analysis was performed using SPSS 17.0 software. The results are expressed as the mean \pm SD of three independent experiments. For each experiment, the statistical analysis was repeated three times. Statistical analysis of differences was performed by one-way analysis of variance (ANOVA) or Student's t-test. ${ }^{*} \mathrm{P}<0.05$ and ${ }^{* *} \mathrm{P}<0.01$ was considered to indicate a statistically significant difference.

\section{Results}

Expression of PAX6 and miR-335 in glioma tissues. The expression of PAX6 was examined in human glioma tissues and normal brain tissues by real-time RT-PCR. As shown in Fig. 1A, the mRNA expression level of PAX6 in glioma tissues was reduced compared with normal brain tissues. Furthermore, the glioma tissues with different grades demonstrated different expression levels of PAX6, the expression of which was the lowest in grade WHO IV, and highest in grade WHO I, which was further confirmed by the data from western blotting. The expression of miR-335 in human glioma tissues and normal brain tissues was then examined. As shown in Fig. 1B, the level of miR-335 in glioma tissues gradually increased, when compared with those in normal brain tissues and its expression was positively associated with the malignancy of gliomas.

PAX6 is a novel target of miR-335. As shown in Fig. 2A, bioinformatical analysis results demonstrated that the putative binding site for miR-335 at the 3'UTR of PAX6 is conserved and the mutant type 3'-UTR of PAX6, which was constructed, was also shown. The results of the luciferase reporter assay 

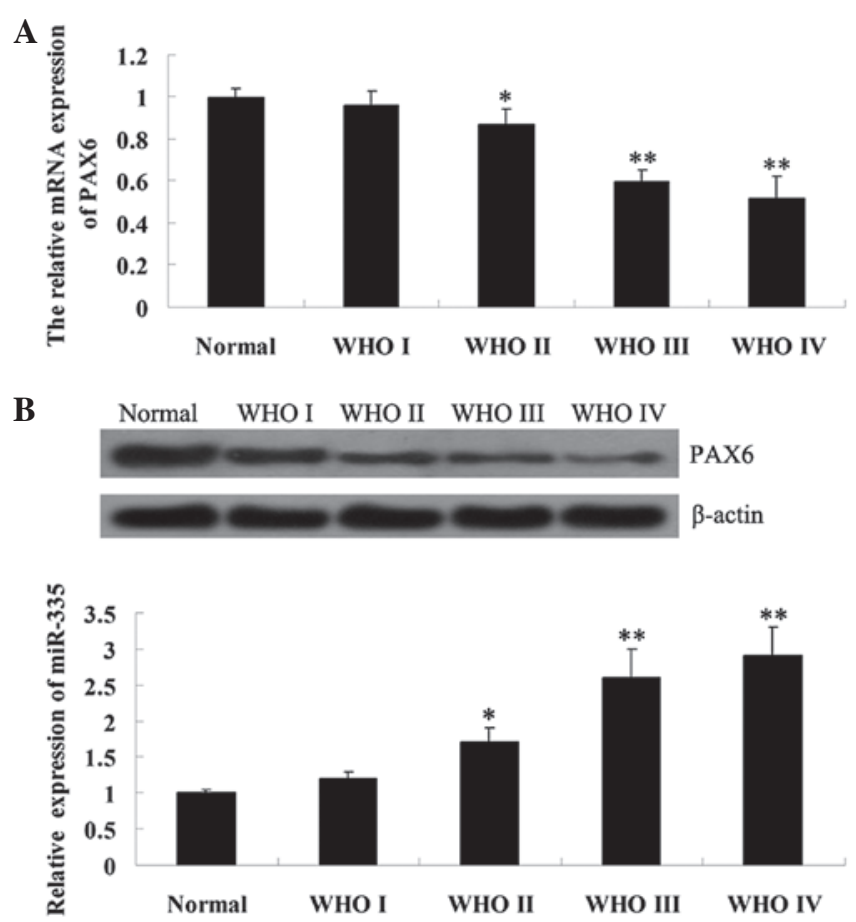

Figure 1. (A) Real-time RT-PCR and western blot analysis were performed to determine the mRNA and protein expression of PAX6 in normal brain tissues (normal) as well as glioma tissues of different grades, respectively ${ }^{* *} \mathrm{P}<0.01$ vs. normal. (B) Real-time RT-PCR was used to examine the relative expression of miR-335 in normal brain tissues (normal) and glioma tissues of different grades, respectively. ${ }^{*} \mathrm{P}<0.05$ vs. normal; ${ }^{* *} \mathrm{P}<0.01$ vs. normal. PAX6, paired box 6; miR-335, microRNA-335

demonstrated that the renilla/firefly value of luciferase activity was markedly decreased only following co-transfection with the wild type 3'UTR of PAX6 and miR-335 in U87 and U251 cells $(\mathrm{P}<0.01)$, whereas the renilla/firefly value of luciferase activity in other groups demonstrated no difference (Fig. 2B). Thus, the present study suggests that PAX6 is a direct target of miR-335 in glioma U87 and U251 cells.

Effect of miR-335 on the protein expression of PAX6. The effect of miR-335 on the protein expression of PAX6 in U87 and U251 cells was further investigated. Following transfection of U87 and U251 cells with an miR-335 mimic or inhibitor, respectively, the miR-335 expression level was examined using real-time RT-PCR. As shown in Fig. 3A, the expression level of miR-335 was significantly upregulated following transfection with the miR-335 mimic, however, it was downregulated following transfection with the miR-335 inhibitor, when compared with that in the control group $(\mathrm{P}<0.01)$. Thus, the transfection efficiency was satisfactory. The protein expression of PAX6 was then determined using western blot analysis and the PAX6 protein level was revealed to be decreased in miR-335-overexpressed cells, however, it was increased in miR-335-downregulated cells (Fig. 3B). According to these data, the present study suggests that miR-335 negatively regulates the expression of PAX6 at a post-transcriptional level in glioma U87 and U251 cells.

Effects of PAX6 and miR-335 on the regulation of glioma cell proliferation. In order to investigate the functions of PAX6
A

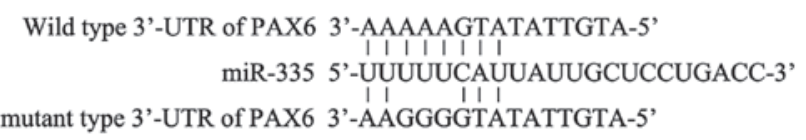

@ wild type ROCK1 3'-UTR $\square$ mutant type ROCK1 3'-UTR

B

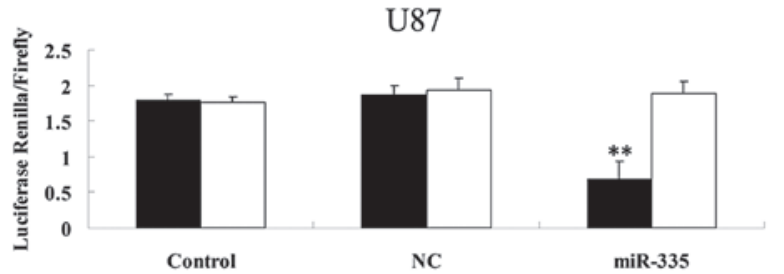

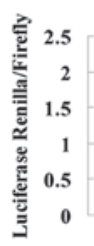

$\mathrm{U} 251$

Figure 2. (A) Bioinformatical analysis demonstrated the putative target sequence of miR-335 in the wild type 3'-UTR of PAX6. For the luciferase reporter assay, a mutant type 3'-UTR of PAX6 with a substitution of AAA to GGG within the putative target sequence. (B) Luciferase assay was performed to determine whether PAX6 was a target of miR-335. A wild type and a mutant type 3'-UTR of PAX6 was subcloned into the psiCHECK ${ }^{\mathrm{TM}} 2$ reporter vector, respectively. PsiCHECK ${ }^{\mathrm{TM}}$ 2-PAX6-3'-UTR or psiCHECK $^{\mathrm{TM}} 2$-mut PAX6-3'-UTR vector plus $50 \mathrm{nM}$ of miR-335 were co-transfected into U87 and U251 cells, respectively. Control: cells without transfection of miRNA; NC: cells transfected with NC miRNA. ${ }^{* *} \mathrm{P}<0.01$ vs. control. PAX6, paired box 6 ; miR-335, microRNA-335; NC, negative control; 3'-UTR, 3'-untranslated region.

and miR-335 in glioma cells, U251 cells were transfected with PAX6-pCMV-NEO-BAM, the miR-335 mimic or the two combined, respectively. As shown in Fig. 4A, the protein expression of PAX6 was markedly upregulated in U251 cells transfected with PAX6-pCMVp-NEO-BAM. Furthermore, the miR-335 mimic inhibited the PAX6 protein expression, however, in the co-transfection group, the PAX6 protein level was restored. Following that, the cell proliferation rate was markedly reduced in PAX6-overexpressed glioma cells compared with control groups, respectively. However, in miR-335-overexpressed U251 cells, the cell proliferation was significantly upregulated, which was effectively reversed by co-transfection with PAX6-pCMV-NEO-BAM and the miR-335 mimic. Taken, together, these results indicate that PAX6 has an inhibitory effect on U251 and U87 cell proliferation, while miR-335 is able to promote glioma cell proliferation.

Effects of PAX6 and miR-335 on the regulation of colony formation in glioma cells. The effects of PAX6 and miR-335 overexpression on colony formation ability in U251 cells were further studied. As shown in Fig. 5, the PAX6 overexpressed U251 cells demonstrated lower colony formation efficiency compared with the control group. However, miR-335 upregulation promoted colony formation in U251 cells, which was reversed by co-transfection with PAX6 and the miR-335 mimic. Accordingly, our findings suggest that PAX6, negatively regulated by miR-335, inhibits the malignant characteristics of glioma cells. 
A

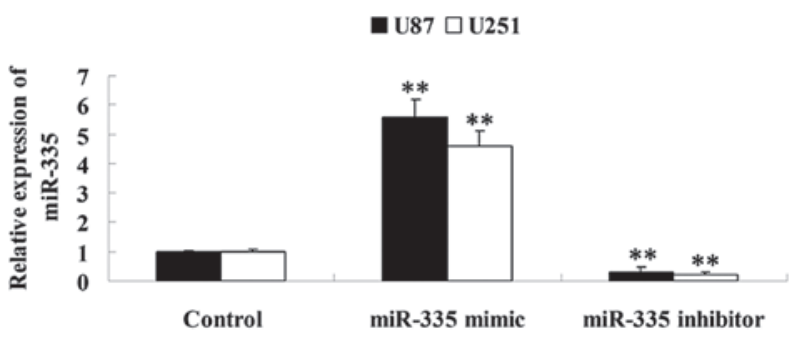

B
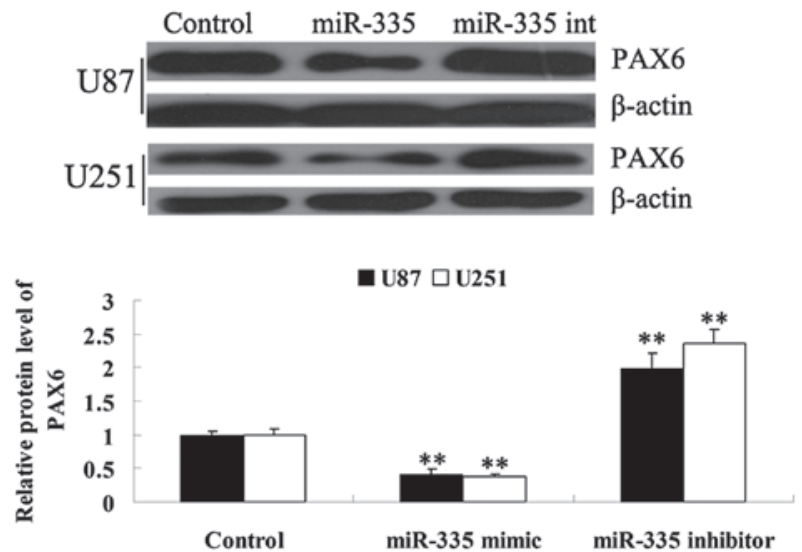

Figure 3. (A) Real-time RT-PCR was used to examine the relative expression of miR-335 in U87 and U251 cells following transfection with the miR-335 mimic or miR-335 inhibitor, respectively. Control: cells without any transfection. ${ }^{* *} \mathrm{P}<0.01$ vs. control. (B) Western blot analysis was performed to determine the protein expression of PAX6 in U87 and U251 cells following transfection with the miR-335 mimic or miR-335 inhibitor, respectively $\beta$-actin was used as an internal control. Control: cells without any transfec tion. ${ }^{* *} \mathrm{P}<0.01$ vs. control. PAX6, paired box 6; miR-335, microRNA-335.
A

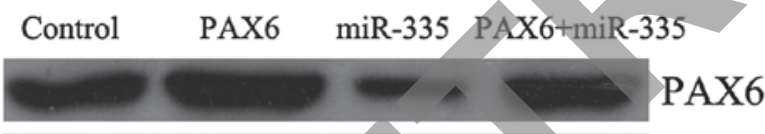

$$
\text { }
$$
$\beta$-actin

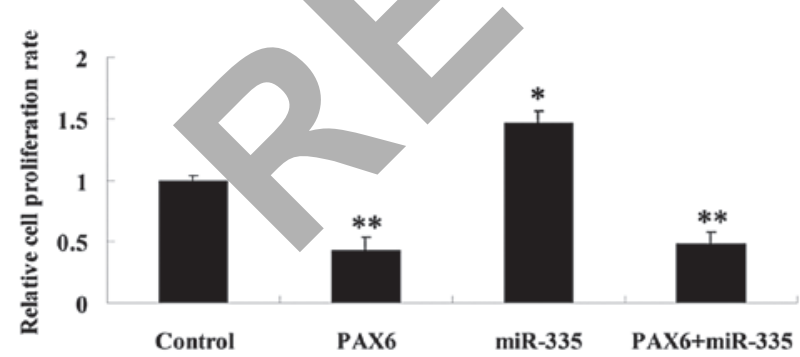

Figure 4. (A) Western blot analysis was performed to determine the protein expression of PAX6 in U251 cells following transfection with PAX6-pCMVp-NEO-BAM, the miR-335 mimic or the two combined, respectively. $\beta$-actin was used as an internal control. Control: cells without any transfection. (B) Cell proliferation assay was used to determine the cell number of U251 cells following transfection with PAX6-pCMVp-NEO-BAM, the miR-335 mimic or the two combined, respectively. Control: cells without any transfection. ${ }^{*} \mathrm{P}<0.05$ vs. control. ${ }^{* *} \mathrm{P}<0.01$ vs. control. PAX6, paired box 6; miR-335, microRNA-335.

Effects of PAX6 and miR-335 on the regulation of glioma cell invasion. The role of PAX6 and miR-335 in the regulation of U251 cell invasion was further investigated. As demonstrated in Fig. 6A, overexpression of PAX6 significantly inhibited $\mathrm{U} 251$ cell invasion $(\mathrm{P}<0.05)$. However, miR-335 overexpression

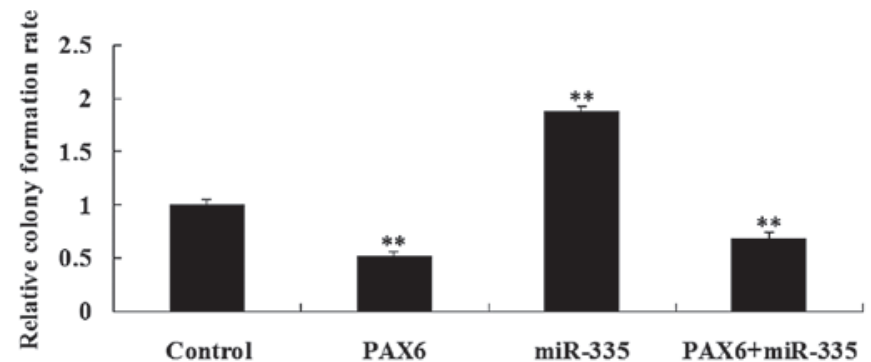

Figure 5. Colony formation assay was used to examine the relative colony formation rate of U251 cells following transfection with PAX6-pCMVp-NEO-BAM, the miR-335 mimic or the two combined, respectively. Control: cells without any transfection. ${ }^{* *} \mathrm{P}<0.01$ vs. control. PAX6, paired box 6; miR-335, microRNA-335.
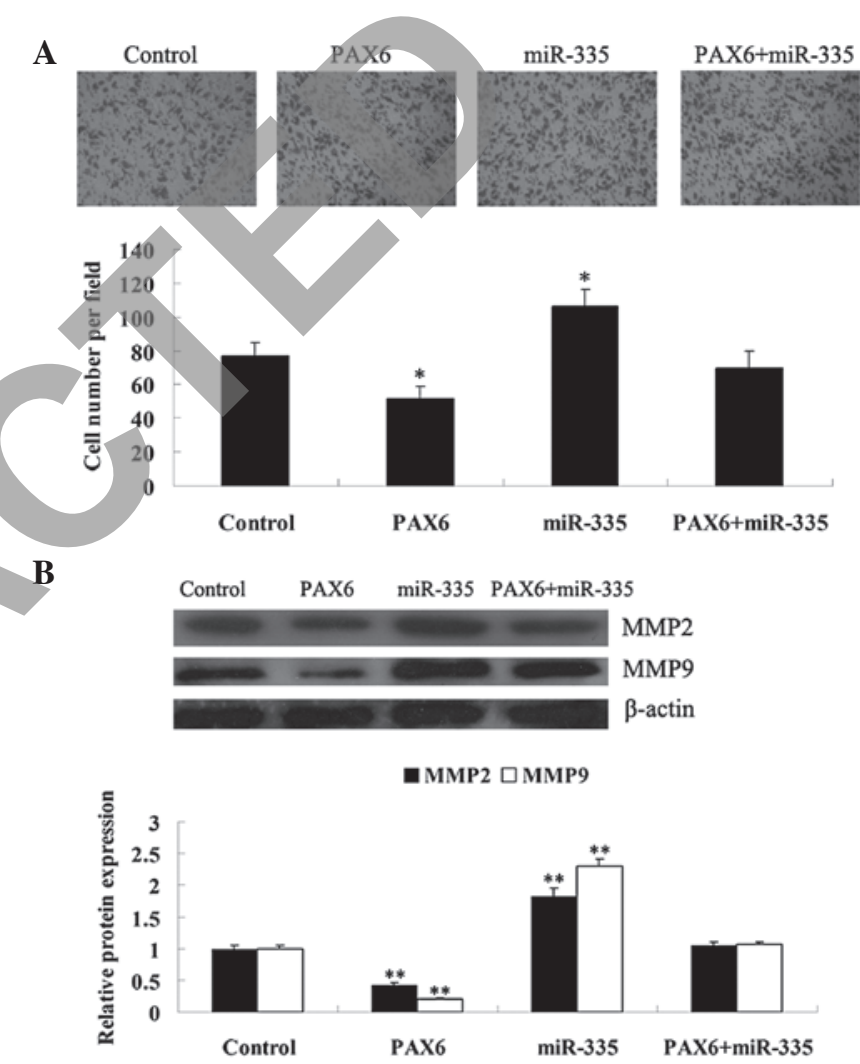

Figure 6. (A) Cell invasion assay was performed to determine the invasion ability of U251 cells following transfection with PAX6-pCMVp-NEO-BAM, the miR-335 mimic or the two combined, respectively. Control: cells without any transfection. ${ }^{*} \mathrm{P}<0.05$ vs. control. (B) Western blot analysis was performed to determine the protein expression of MMP-2 and MMP-9 in U251 cells following transfection with PAX6-pCMVp-NEO-BAM, the miR-335 mimic or the two combined, respectively. $\beta$-actin was used as an internal control. Control: cells without any transfection. ${ }^{* *} \mathrm{P}<0.01$ vs. control. PAX6, paired box 6; miR-335, microRNA-335; MMP, matrix metalloproteinase.

notably promoted cell invasion $(\mathrm{P}<0.05)$, which was effectively reversed by PAX6 overexpression. These data indicate that PAX6 has an inhibitory effect on glioma cell invasion, while miR-335 is able to promote it.

MMP-2 and MMP-9 are crucial in the regulation of cancer cell invasion. Thus, the alterations of MMP-2 and MMP-9 expression were further investigated, following transfection of U251 cells with PAX6-pCMV-NEO-BAM, the miR-335 mimic or the two combined, respectively. As shown in Fig. 6B, the protein expression of MMP-2 and MMP-9 were decreased 
in PAX6-overexpressed U251 cells, when compared with that in the control group $(\mathrm{P}<0.01)$. However, their protein levels were upregulated following transfection with the miR-335 mimic $(\mathrm{P}<0.01)$, which was reversed by the overexpression of PAX6.

Taken together, our findings suggest that PAX6, negatively regulated by miR-335, is able to inhibit MMP-2 and MMP-9 expression, which is possibly responsible for the suppressive effect of PAX6 on glioma cell invasion.

\section{Discussion}

As one of the most common types of primary brain cancer, the tumorigenesis of glioma is regulated by a complex cascade of molecular events monitoring cell proliferation and motility (8). Thus, understanding the molecular regulatory mechanisms has vital significance. The present study demonstrated that the expression of PAX6 was reduced parallel to the upregulation of miR-335. More importantly, the present study for the first time, to the best of our knowledge, identified PAX6 as a direct target of miR-335. Functional analysis revealed that PAX6, negatively regulated by miR-335, effectively inhibited cell proliferation, colony formation and invasion in glioma cells.

As a key transcription factor, PAX6 has been demonstrated to be involved in the development of the eye, central nervous system and pancreas $(3,24)$. Previously, PAX6 was revealed to be involved in the regulation of malignant glioma $(4,25)$. Zhou et al demonstrated a correlation between a low expres sion level of PAX6 and unfavorable patient outcomes in patients with malignant astrocytic gliomas (5). The present study also demonstrated that the expression of PAX6 was downregulated in glioma tissues compared with normal brain tissues. The authors further demonstrated that PAX6 acted as a tumor suppressor in glioblastoma cells and the overexpression of PAX6 was able to effectively inhibit the growth of glioblastoma cells in vitro and in vivo (26), which is partially consistent with our findings. Despite the suppressive effect of PAX6 on glioma being revealed, the regulatory mechanisms remain largely unknown.

Based on bioinformatical predication, a luciferase reporter assay was performed and PAX6 was identified as a novel target of miR-335, which has been demonstrated to be implicated in various types of cancer (14-22). However, the detailed role of miR-335 in the development and progression of various types of cancer remains controversial. For instance, it has been suggested that miR-335 may function as a tumor suppressor in osteosarcoma (14), however, it may act as an oncogene in meningiomas (21). These findings suggest that the exact regulatory function of miR-335 in tumorigenesis is tissue specific, since different microenvironments in various types of cancer may have different effects on the expression and activity of miR-335.

Previously, miR-335 was revealed to be involved in several types of malignant tumor in the central nervous system $(23,27,28)$. Shu et al reported that miR-335 acted as a promoter in growth and invasion in malignant astrocytoma, partially at least through directly targeting Daam1 (27). In addition, the authors further demonstrated that miR-335 was required for the differentiation of malignant glioma cells induced by the activation of the $\mathrm{cAMP} /$ protein kinase $\mathrm{A}$ pathway (28). Jiang et al demonstrated that the upregulated expression of miR-335 was associated with advanced tumor progression as well as a poorer survival time in patients with malignant glioma (23), indicating that miR-335 may become an independent marker for the predication of the clinical outcome of patients with gliomas. Consistently with their findings, the present study also revealed that a high expression of miR-335 was significantly associated with a higher WHO grade of glioma.

Since the present study for the first time, to the best of our knowledge, identified PAX6 as a target of miR-335, their association in glioma cells was further determined. As expected, miR-335 overexpression significantly downregulated the protein expression of PAX6, while inhibition of miR-335 promoted the protein level of PAX6 in U87 and U251 cells, which are WHO IV grade. In fact, several other miRNAs have also been reported to directly target PAX6, including miR-290-295, miR-365-3p and miR-328 (29-31). Huang et al demonstrated that miR-223 promoted the growth and invasion of glioblastoma cells by targeting PAX6 (32). Since our findings revealed a novel regulatory pathway involved in PAX6 and miR-335, the present study enriches the understanding of the molecular mechanisms by which PAX6 is regulated in glioma.

In addition, the present study demonstrated that the protein levels of MMP-2 and MMP-9 were decreased in the PAX6-overexpressed glioma cells, however, were upregulated in the miR-335-overexpressed glioma cells. MMP-2 and MMP-9 are two key proteases secreted by tumor and microenvironmental cells and are able to lead to extracellular matrix degradation, which directly links to cancer cell invasion and metastasis (33). Furthermore, PAX6 has been reported to suppress the transcription of the MMP-2 gene (4). Based on these findings and ours, the present study suggests that miR-335 promotes glioma cell invasion, possibly through directly inhibiting PAX6 and thus upregulating MMP-2 and MMP-9.

In conclusion, the present study demonstrated that PAX6, as a novel target of miR-335, functions as a tumor suppressor in malignant glioma cells. Our findings suggest that PAX6 and miR-335 are promising therapeutic targets for glioma.

\section{Acknowledgements}

This study was supported by Hunan Provincial Innovation Foundation For Postgraduate.

\section{References}

1. Shaham O, Menuchin Y, Farhy C and Ashery-Padan R: Pax6: a multi-level regulator of ocular development. Prog Retin Eye Res 31: 351-376, 2012.

2. Gosmain Y, Cheyssac C, Heddad Masson M, Dibner C and Philippe J: Glucagon gene expression in the endocrine pancreas: the role of the transcription factor Pax6 in alpha-cell differentiation, glucagon biosynthesis and secretion. Diabetes Obes Metab 13 (Suppl 1): 31-38, 2011.

3. Xie Q, Yang Y, Huang J, et al: Pax6 interactions with chromatin and identification of its novel direct target genes in lens and forebrain. PLoS One 8: e54507, 2013.

4. Mayes DA, Hu Y, Teng Y, et al: PAX6 suppresses the invasiveness of glioblastoma cells and the expression of the matrix metalloproteinase-2 gene. Cancer Res 66: 9809-9817, 2006. 
5. Zhou YH, Tan F, Hess KR and Yung WK: The expression of PAX6, PTEN, vascular endothelial growth factor, and epidermal growth factor receptor in gliomas: relationship to tumor grade and survival. Clin Cancer Res 9: 3369-3375, 2003.

6. Stewart LA: Chemotherapy in adult high-grade glioma: a systematic review and meta-analysis of individual patient data from 12 randomised trials. Lancet 359: 1011-1018, 2002.

7. Zhu VF, Yang J, Lebrun DG and Li M: Understanding the role of cytokines in Glioblastoma Multiforme pathogenesis. Cancer Lett 316: 139-150, 2012

8. Sathornsumetee S, Reardon DA, Desjardins A, Quinn JA, Vredenburgh JJ and Rich JN: Molecularly targeted therapy for malignant glioma. Cancer 110: 13-24, 2007.

9. Pulkkanen KJ and Yla-Herttuala S: Gene therapy for malignant glioma: current clinical status. Mol Ther 12: 585-598, 2005.

10. Ambros V: The functions of animal microRNAs. Nature 431: $350-355,2004$

11. Nikaki A, Piperi $\mathrm{C}$ and Papavassiliou AG: Role of microRNAs in gliomagenesis: targeting miRNAs in glioblastoma multiforme therapy. Expert Opin Investig Drugs 21: 1475-1488, 2012

12. Ling N, Gu J, Lei Z, et al: microRNA-155 regulates cell proliferation and invasion by targeting FOXO3a in glioma. Oncol Rep 30: 2111-2118, 2013.

13. Lin L, Chen X, Peng X, et al: MicroRNA-128 promotes cell-cell adhesion in U87 glioma cells via regulation of EphB2. Oncol Rep 30: 1239-48, 2013

14. Wang Y, Zhao W and Fu Q: miR-335 suppresses migration and invasion by targeting ROCK1 in osteosarcoma cells. Mol Cell Biochem 384: 105-11, 2013

15. Gong M, Ma J, Guillemette R, et al: miR-335 inhibits small cell lung cancer bone metastases via IGF-1R and RANKL pathways. Mol Cancer Res: Aug 21,2013 (Epub ahead of print).

16. Walter BA, Valera VA, Pinto PA and Merino MJ: Comprehensive microRNA profiling of prostate cancer. J Cancer 4: 350-357, 2013.

17. Cao J, Cai J, Huang D, et al: miR-335 represents an invasion suppressor gene in ovarian cancer by targeting Bcl-w. Onco Rep 30: 701-706, 2013.

18. Xiong SW, Lin TX, Xu KW, et al: MicroRNA-335 acts as candidate tumor suppressor in prostate cancer. Pathol Oncol Res 19: 529-537, 2013

19. Liu J, Mao Q, Liu Y, Hao X, Zhang S and Zhang J: Analysis of miR-205 and miR-155 expression in the blood of breast cancer patients. Chin J Cancer Res 25: 46-54, 2013.
20. Dohi O, Yasui K, Gen Y, et al: Epigenetic silencing of miR-335 and its host gene MEST in hepatocellular carcinoma. Int J Oncol 42: 411-418, 2013.

21. Shi L, Jiang D, Sun G, et al: miR-335 promotes cell proliferation by directly targeting Rb1 in meningiomas. J Neurooncol 110 $155-162,2012$

22. Yan Z, Xiong Y, Xu W, et al: Identification of hsa-miR-335 as a prognostic signature in gastric cancer. PLoS One 7: e40037, 2012.

23. Jiang J, Sun X, Wang W, et al: Tumor microRNA-335 expression is associated with poor prognosis in human glioma. Med Oncol 29: 3472-3477, 2012.

24. Yamaoka T and Itakura M: Development of pancreatic islets (review). Int J Mol Med 3: 247-261, 1999.

25. Liu RZ, Monckton EA and Godbout R: Regulation of the FABP7 gene by PAX6 in malignant glioma cells. Biochem Biophys Res Commun 422: 482-487, 2012.

26. Zhou YH, Wu X, Tan F, et al: PAX6 suppresses growth of human glioblastoma cells. J Neurooncol 71: 223-229, 2005.

27. Shu M, Zheng X, Wu S, et al: Targeting oncogenic miR-335 inhibits growth and invasion of malignant astrocytoma cells. Mol Cancer 10: 59, 2011.

28. Shu M, Zhou Y, Zhu W, et al: MicroRNA 335 is required for differentiation of malignant glioma cells induced by activation of cAMP/protein kinase A pathway. Mol Pharmacol 81: 292-298, 2012

29. Kaspi H, Chapnik E, Levy M, Beck G, Hornstein E and Soen Y: Brief report: miR-290-295 regulate embryonic stem cell differentiation propensities by repressing Pax6. Stem Cells 31: 2266-2272, 2013.

30. Wang J, Wang X, Wu G, Hou D and Hu Q: MiR-365b-3p, down-regulated in retinoblastoma, regulates cell cycle progression and apoptosis of human retinoblastoma cells by targeting PAX6. FEBS Lett 587: 1779-1786, 2013.

31. Chen KC, Hsi E, Hu CY, Chou WW, Liang CL and Juo SH: MicroRNA-328 may influence myopia development by mediating the PAX6 gene. Invest Ophthalmol Vis Sci 53: 2732-2739, 2012.

32. Huang BS, Luo QZ, Han Y, Li XB, Cao LJ and Wu LX: microRNA-223 promotes the growth and invasion of glioblastoma cells by targeting tumor suppressor PAX6. Oncol Rep 30: 2263-2269, 2013.

33. Levicar N, Nuttall RK and Lah TT: Proteases in brain tumour progression. Acta Neurochir (Wien) 145: 825-838, 2003. 